\title{
FRAME: An Innovative Incentive Scheme in Vehicular Networks
}

\author{
Feng Li and Jie Wu \\ Department of Computer Science and Engineering \\ Florida Atlantic University \\ Boca Raton, FL 33431
}

\begin{abstract}
Vehicular ad hoc networks (VANETs) are envisioned to provide promising applications and services. One critical deployment issue in VANETs is to motivate vehicles and their drivers to cooperate and contribute to packet forwarding in vehicle-to-vehicle or vehicle-to-roadside communication. In this paper, we examine this problem, analyze the drawbacks of two straightforward schemes, and present a secure incentive scheme to stimulate cooperation and contribution in VANETs. We first define the measurement of contribution according to the unique characteristics of VANET communication. Our scheme uses the weighted rewarding component to ensure fairness.
\end{abstract}

\section{INTRODUCTION}

In the near future, most new vehicles will be equipped with short range radios and a powerful processing device [1]. Therefore, the vehicles will be able to communicate with each other via the high throughput short range radio. By utilizing the communication and processing capabilities of the vehicles, vehicular ad hoc networks (VANETs) will contribute to safer and more efficient roads by providing timely information to drivers and concerned authorities.

Similar to other mobile ad hoc networks (MANETs), vehicular networks are self-organizing and are formed directly by a set of smart vehicles. To achieve the network's designed functionality, individual vehicles need to cooperate in packet forwarding in vehicle-to-vehicle communication. However, some selfish users in the vehicles may not want to forward the packets if it will not benefit them in some way. Moreover, malicious users can also utilize the forwarding behavior to launch attacks if there is no cost. To bring the vehicular networks to their full potential, an incentive scheme needs to be developed and employed according to the VANETs' unique features and potential applications to stimulate cooperation.

Fig. 1 shows a typical packet forwarding process in VANETs. Vehicles encounter one another at different times, and packets are opportunistically forwarded. If an intermediate vehicle stores a packet for a long time or actively sprays the packet to other vehicles, the packet will be more likely to reach the intended destination. Therefore, by combining the storage time and number of sprays, we define our measurement of contribution for the intermediate vehicles. To stimulate intermediate vehicles to contribute more, the source vehicle should follow the incentive scheme and reward the intermediate vehicles according to their measured contributions.

The design of the reward calculation is the core of the incentive scheme. It should guide users to follow the protocols

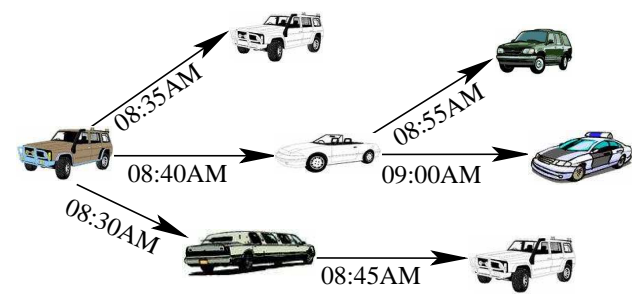

Fig. 1. An example vehicular ad hoc network (VANET).

in the VANET and encourage vehicles' desirable behavior. We first examine two intuitive schemes: receipt counting and proportional rewarding. Both of them have some of the desirable properties as well as some unavoidable issues. In an effort to achieve fairness and provide motivative stimulation for participation, we propose a Fair Reimbursement And Motivating swEepstake scheme (FRAME).

The FRAME scheme contains two major components: a weighted rewarding component and a sweepstake component. Based on the contribution measurement, the weighted rewarding component utilizes a convex function to calculate each vehicle's weight and allocate rewards according to the calculated weights. The sweepstake component awards a fixed amount to the winning vehicle that participates in the forwarding. Each vehicle has a designed probability to be the winner. The design of the winning probability keeps the packet forwarding attractive to the potential intermediate vehicles. Otherwise the potential users in the vehicles may not want to participate in the communication, and the VANET applications cannot succeed.

The contributions of this paper are three-fold. First, we propose a promising weighted rewarding scheme which is designed based on the unique characteristics of VANETs. Second, we introduce a sweepstake method in the incentive scheme which can attract more nodes to participate in forwarding. Third, we discuss the desirable properties for the incentive schemes in VANETs and illustrate why the FRAME scheme satisfies these properties.

\section{RELATED WORK}

To stimulate cooperation among selfish nodes in ad hoc networks, several incentive schemes have been proposed [2], [3], [4], [5], [6], [7]. Buttyán and Hubaux brought the virtual 
Table I List of notations

\begin{tabular}{|c|c|}
\hline$P K / R K$ & Public key / private key \\
\hline$E(*) / H(*)$ & Encryption using private key / hash \\
\hline$T / \Delta T$ & Time stamp / time duration \\
\hline$K$ & Number of sprays \\
\hline$C / C_{T}$ & Contribution / total contribution of the tree \\
\hline$V / V_{r} / V_{s}$ & Reward for total / rewarding / sweepstake \\
\hline$Q / P$ & Proportion in $V_{r} /$ probability to win $V_{s}$ \\
\hline$i, j, l$ & Vehicles $i, j, l$ \\
\hline$s u b(i) / c h d(i)$ & Subtree rooted at $i /$ Children set of $i$ \\
\hline
\end{tabular}

credit into incentive schemes to stimulate packet forwarding in [8]. Nodes are willing to help in forwarding others' packets to earn the virtual credit, which can be used when they need to send their own packets. In [9], Zhong et al. presented an incentive scheme that utilizes the VCG mechanism to select the best available single path. Lee et al. discussed some unique characteristics of the incentive schemes for VANETs in [2] and proposed a receipt counting reward scheme, which focuses on the incentive for spraying. We propose a more comprehensive weighted rewarding method in this paper and compare it with the receipt counting scheme.

Based on the previous works related to the lottery theory [10], Douceur and Moscibroda designed three different lottery schemes to encourage contribution in [4], as well as a set of properties to evaluate a lottery scheme. However, in all the lottery schemes in [4], only one winner will be probabilistically selected and receive all the reward. Although fairness is considered in the design of winning probability, nodes other than the winner receives nothing. This will deter the inherently conservative users to participate. We introduce a sweepstake component in this paper, which is inspired by [4], to provide a probabilistic reward to attract vehicles to participate.

\section{Preliminary: Security Entities}

VANETs should be developed based on the existing infrastructure of the traffic system [11], [12]. We list and propose some feasible security entities to serve as the building blocks for the secure incentive scheme FRAME.

Certificate authority (CA). Since the vehicles will have abundant computational power, most security applications in VANETs will rely on the PKI-based strong authentication. The certificate authority (CA) should be commonly trusted government agencies, such as the Department of Motor Vehicles (DMV), which authenticate the vehicle with some outof-band mechanism when issuing the certificate. The CA can issue a digital driver license or digital plate [13], which is a certificate that bears the CA's signature to prove the identity of the vehicle or the driver in the vehicle.

Forwarding administration authority (FA). The forwarding administration authority (FA), which should also be a trusted third party, authorizes the forwarding of a packet and administrates the charge/reward process.

The forwarding administration authority contains several different parts: authorization and charging points, evidence collection points, an incentive processing part, and rewarding centers. The authorization and charging points can be some roadside storage points. They authenticate the source vehicle for one round of packet forwarding, and charge virtual credit from the vehicle's account as the promised incentive. The evidence collection points are also roadside storage points that collect evidence of contribution from vehicles. The incentive calculation part has wired connection with other parts. It follows the incentive scheme to calculate the rewards for every participating vehicle. Gas stations or grocery stores could be the rewarding centers, where the drivers can redeem their virtual rewards from their account.

For the FRAME scheme, the timing evidence is crucial when calculating the contribution. One possible approach is to employ a tamper-proof hardware which securely provide a time-stamp on a packet for current time.

\section{FRAME SCHEME}

Based on the security settings, we propose the core of the FRAME scheme in this section.

We use a forwarding tree to represent the propagation process of a packet $M$. The root represents the source vehicle. Each intermediate vehicle corresponds to one node in the tree. The parent node corresponds to the vehicle that first sprays $M$ to the current node. The descendant nodes correspond to the vehicles that receive packets from the current node. Vehicles will ignore a packet if they have received it before. Therefore, each node will appear only once in the tree. Each link in the tree corresponds to an encounter in the vehicle network, which is associated with the time stamp of the encounter. Also note that for unicast scenarios, only one leaf will be the destination node. We will use the terms node and vehicle interchangeably in the following sections.

\section{A. Contribution measurement}

In the VANETs, if all vehicles store a packet longer and propagate the packet to more vehicles, the probability that the destination will get the packet increases. Therefore, an intermediate vehicle's contribution of relaying a packet contains the following aspects: the time period that it stores the packet, $\Delta T_{i}$, and the number of direct sprays that it makes, $K_{i}$. The measurement of contribution, $C$, for an intermediate vehicle $i$ can be calculated as:

$$
C_{i}=\alpha \cdot \Delta T_{i}+(1-\alpha) \cdot K_{i}
$$

where $\alpha$ is the factor to balance between the two metrics.

$K_{i}$ and $\Delta T_{i}$ are calculated based on the secured evidences. We give the details on how to secure these evidences in subsection IV-E. There are four phases in a packet forwarding process. In the first phase, the source vehicle should interact with the forwarding administration authority to get the signature as permission to forward the packet.

In the second phase, the source vehicle will present the signature and spray the packet to each vehicle that it encounters. A vehicle $i$, which receives the packet, will first get a timestamp with the signature of the sender as the proof of the time 
the storage was initiated. Vehicle $i$ can then spray the packet to others. For each spray, vehicle $i$ should require a receipt. If vehicle $i$ decides to discard the packet, it needs to get another time-stamp from its temper-proof component.

After the packet reaches the destination, the third phase starts. Vehicles need to surrender all the receipts and the time-stamps with the signatures to the evidence collection and charging points within a pre-specified period of time. If they still store the packet, the end of storage time will be set to the time that the packet reaches the destination.

In the forth phase, the $F A$ constructs the tree. For a node $i, K_{i}$ is equal to the number of valid receipts that vehicle $i$ collected, and $\Delta T_{i}$ is calculated based on the time-stamps of the start and end of storage time. Some nodes may be missing in the tree, as some vehicles may miss the third phase. This is allowed in the contribution calculation.

\section{B. Two straightforward schemes}

We will first examine two intuitive incentive methods which are used in previous research works [2], [4], [5], [6].

Receipt counting method. In this method, when a vehicle $i$ forwards a packet to another vehicle $j, j$ should give $i$ a receipt of this packet if it has never received the packet before. The source of the packet should promise a fixed value for each receipt. All the forwarding nodes can claim a reward based the number of receipts in their hand. If one intermediate node $i$ holds $K_{i}$ receipts, the total reward that vehicle $i$ will receive is $K_{i}$ times each receipt's value.

This method is adapted in [2]. It is very flexible since nodes are allowed to redeem the receipts they have before the packet reaches the destination. However, this method creates an overspending problem for the source. The source vehicle cannot know or control the total amount of reward since the number of nodes in the tree cannot be predicted.

Fig. 2(a) illustrates an example using this method. Node $i$ collects 2 receipts. If each receipt's value equals 1 , node $i$ will get 2 virtual credits as the reward.

Proportional rewarding method. One direct way to solve the overspending problem is to fix the total amount of reward and compensate forwarding nodes according to their proportional contribution. In this proportional rewarding method, the source will be charged a fixed amount of total reward $V$ before it sends out a packet. After the packet reaches the destination, each node $i$ that participated in the forwarding should report its contribution $C_{i}$ to the evidence collection points. The total amount of contribution of the tree is then calculated as $C_{T}=$ $\sum_{i \in \text { tree }} C_{i}$. Each node $i$ that participated in the forwarding will be credited $V \cdot \frac{C_{i}}{C_{T}}$ as the reward of the forwarding.

Proportional rewarding guarantees fairness and solves the overspending problem. However, the problem with this method is that it causes forwarding nodes to prefer keeping rather than spraying when they contribute to the forwarding. That is because if a node $i$ sprays a packet to another node $j$ that is not the destination node, $i$ 's proportion $\frac{C_{i}}{C_{T}}$ may decrease since $j$ can contribute to the system after it receives the packet and $C_{T}$ will be pushed higher by including $C_{j}$.

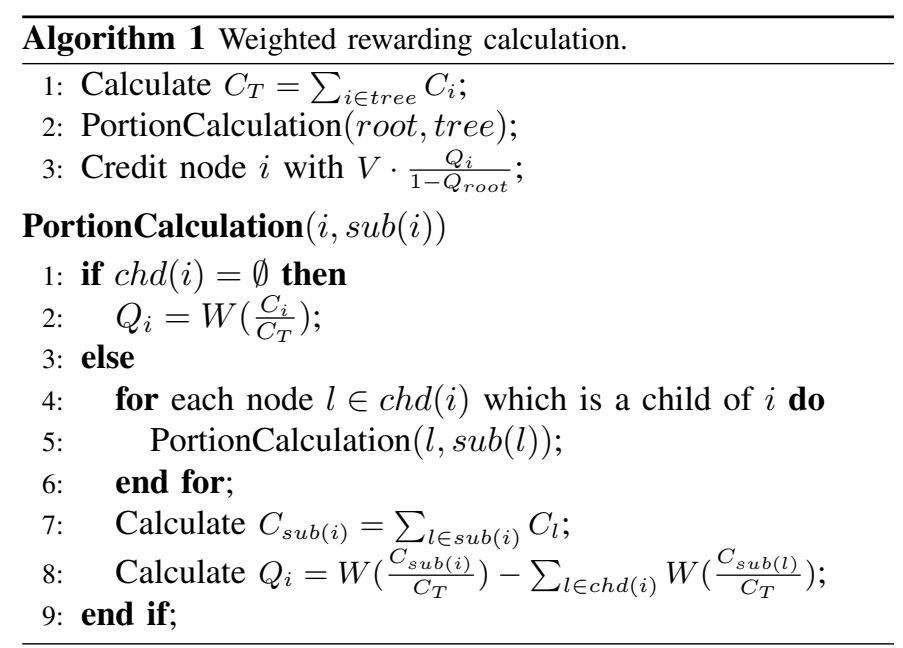

An example for this method is shown in Fig. 2(b). Suppose the source promises 10 as the total reward. Node $i$ contributes: $\frac{0.09 \cdot 47+0.91 \cdot 2}{43.3}=15.4 \%$ of the total contribution. Therefore, node $i$ gets 1.54 as the reward.

\section{Weighted rewarding component}

Since both intuitive schemes have drawbacks, we propose FRAME to address these issues. The FRAME scheme includes two components: a weighted rewarding component, and a sweepstake component.

The source should commit a fixed amount of reward $V_{r}$ for the weighted rewarding. The vehicles that participate in the forwarding will receive a share of the total reward. However, the share is divided to the calculated weight instead of direct contribution value in this method.

In the tree representation, each node sprays the packet to its child nodes. To encourage spraying, we need to link the children's contribution when calculating a node's weight. One intuitive way is to assign the total contribution of each subtree as the weight of each node. However, this gives the child nodes the incentive to bypass the intermediate node and directly connect to the root. Suppose we have two nodes $i$ and $j$. When node $j$ is a child of the root, $j$ 's share is $\frac{W_{j}}{\sum_{l \in \text { tree\& } l \neq i} W_{l}+W_{i}}$. When node $j$ is a child of node $i$, $i$ 's weight will increase to $W_{i}^{\prime}=W_{i}+W_{j}$ and $j$ 's share is $\frac{W_{j}}{\sum_{l \in \text { tree\&l } \neq i} W_{l}+W_{i}+W_{j}}$, which is smaller than in the previous case. Therefore, node $j$ will be reluctant to join the forwarding tree through $i$.

We use $\operatorname{sub}(i)$ to represent the subtree rooted at node $i$, and $C_{s u b(i)}$ to denote the total contribution of all nodes in the subtree, where $C_{s u b(i)}=\sum_{l \in s u b(i)} C_{l}$. We calculate weight $W\left(C_{i}\right)$ by using $C_{i}$ as the input. According to the discussion of the previous methods' drawbacks, the design of the weight should have the following attributes to avoid the problems. For node $i$ :

1) If $C_{i}$ increases to $C_{i}^{\prime}$, we have $W\left(C_{i}^{\prime}\right)>W\left(C_{i}\right)$.

2) If $C_{i}=C_{j}$ and $C_{s u b(i)}>C_{s u b(j)}$, we should have $W\left(C_{i}\right)>W\left(C_{j}\right)$. This attribute promotes competition to spray and recruit descendent nodes. 


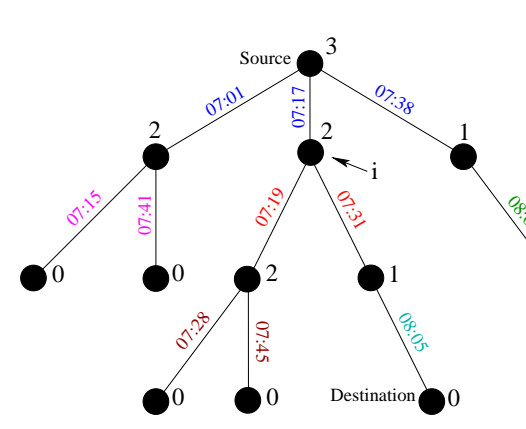

(a) Receipt counting

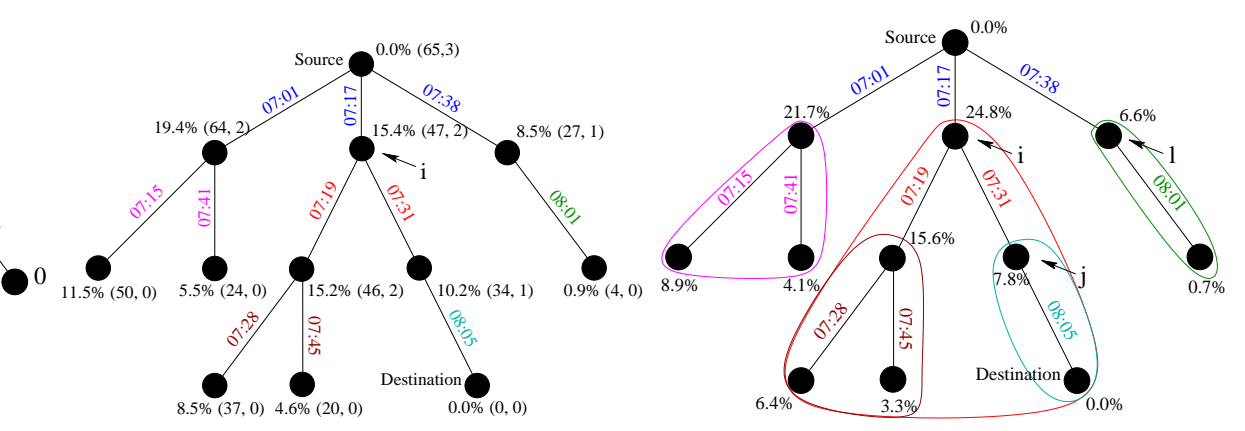

(b) Proportional

(c) Weighted

Fig. 2. Tree representation for three incentive schemes.

3) If $\sum_{l} C_{l}=C_{i}$, we should have $\sum_{l} W\left(C_{l}\right) \leqslant$ $W\left(\sum_{l} C_{l}\right)=W\left(C_{i}\right)$.

We choose a strictly convex function to define our weight which can achieve these attributes. The weight is defined as:

$$
f(x)=a \cdot x^{2}+b \cdot x,
$$

where $a+b=1, a>0$ and $b>0$.

For subtree $\operatorname{sub}(i), W\left(C_{\operatorname{sub}(i)}\right)=f\left(\frac{C_{s u b(i)}}{C_{T}}\right)$. For a node $i$ on the tree, denote the set of $i$ 's children as $\operatorname{chd}(i)$. The portion $Q_{i}$ of $V_{r}$ for node $i$ is calculated as:

$$
Q_{i}=W\left(C_{\text {sub }(i)}\right)-\sum_{l \in \operatorname{chd}(i)} W\left(C_{\text {sub }(l)}\right) .
$$

Notice that a leaf node's $Q_{i}=W\left(\frac{C_{i}}{C_{T}}\right)$, for non-leaf node $Q_{i}$ may not equal $W\left(C_{i}\right)$. For the intermediate vehicle corresponding to node $i$, it can receive $Q_{i} \cdot V_{r}$ as the reward.

When the packet has reached the intended destination, an acknowledgement will be propagated in the VANET and stored in the evidence collection points. The forwarding administration authority will collect the evidence, construct the tree, calculate each node's portion, and credit the nodes according to the calculation. Algorithm 1 presents the calculation process.

In Fig.2(c), node $i$ 's contribution is 6.7. In this scheme, nodes in $\operatorname{sub}(i)$ also contribute to $i$ 's weight. So $i$ will get $24.8 \%$ of the total $V_{r}$. Let us examine another case where $j$ is not a child of $i$ but rather the child of $l$. In this case, $Q_{j}$ remains the same, while $Q_{i}$ is reduced to $19.5 \%$.

One detail that needs to be noticed in algorithm 1 is the portion of $V_{r}$ for the root. However, since the root is the sender of the packet, it should not get a share of $V_{r}$. Therefore, we scale each node's portion to $\frac{Q_{i}}{1-Q_{\text {root }}}$ and assign 0 to the root.

\section{Sweepstake component}

The weight rewarding component guarantees the fairness of our incentive scheme. However, since the value for one packet is limited, the total amount of reward should also be small. The number of nodes that help forward the packet would grow over time. When the forwarding tree grows to a certain size, potential forwarding nodes outside the tree may become reluctant to participate in the forwarding. This is because the existing nodes in the tree already accumulated a large amount

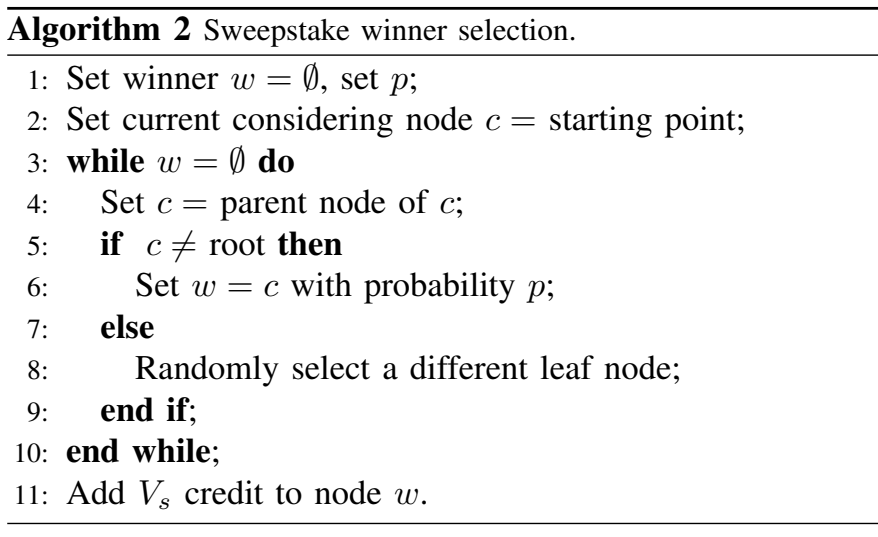

of contribution to the forwarding. Even though the new node joins the tree and takes every effort to forward the packet, it can only get a small portion of the total reward, which is not attractive.

Moreover, the weighted rewarding component treats all the nodes in the tree fairly. However, in the unicast case, only one of the branches in the tree will hit the intended destination. We should provide additional incentives to the "lucky" nodes in that branch of the tree. By doing so, intermediate nodes will make additional efforts to become the lucky nodes besides storing the packet longer and spraying to more nodes. This will improve the VANETs' efficiency.

Based on the prospect theory [14], we introduce a sweepstake component in our FRAME scheme to achieve this additional incentive. One of the intermediate nodes will be chosen probabilistically and awarded with a fixed amount of reward for sweepstake $V_{s}$.

The sweepstake scheme is straightforward. The first step is to select one leaf node on the tree as the starting point. For unicast applications, the intended destination should be selected. For broadcast applications, since all the leaf nodes are intended destinations, one leaf node is randomly selected.

The winner of the sweepstake will be selected from the branch from the starting point to the root. The starting node's parent node $i$ has a probability $P_{i}=p$ to win the sweepstake. Other nodes except $i$ have the probability $1-p$ to win the sweepstake. Among them, $i$ 's parent node $j$ has the probability 
$P_{j}=p \cdot(1-p)$ to win, and $j$ 's parent node has the probability $p \cdot(1-p)^{2}$ to win. Therefore, the sweepstake can be recursively defined until the root has been reached. Since the root node is the source vehicle, it should not participate in the sweepstake. A leaf node which is different from the starting point will be selected and the winner selection will continue on the corresponding branch.

Assume $p=0.4$ and take the example in Fig.2(c). Node $j$ has the probability 0.4 to win $V_{s}$, and node $i$ has the probability 0.24 to win $V_{s}$.

\section{E. Securing the evidences}

We assume that each vehicle in the VANET keeps one original identification certificate. This certificate contains the vehicle's identity (e.g. plate number), the vehicle $i$ 's public key $P K_{i}$ and the certificate authority's signature $E_{C A}\left(H\left(i, P K_{i}\right)\right)$. Each vehicle $i$ should also store its private key $R K_{i}$, the CA's public key $P K_{C A}$, and the forwarding administration authority's public key $P K_{F A}$. At the beginning of any interaction, the security entities will authenticate each other. An entity in the VANET can authenticate another entity $i$ by sending a random number $N$. Entity $i$ should present $P K_{i}$, certificate from $\mathrm{CA}$, and $E_{i}(N)$ as proof of having $R K_{i}$.

When acting as the source of a packet, vehicle $s$ needs to contact one of the authorization and charging points, which represents the forwarding administration authority $F A$. After authentication, $F A$ charges a certain amount of credit $V$ from $s$, and gives the vehicle a signature on the intended packet $M$ as the evidence of approval.

$$
F A \rightarrow s \quad: \quad V, T, E_{F A}(H(M, T))
$$

With this evidence, the source has the right to propagate the packet. This evidence prevents selfish or malicious nodes from propagating unauthorized packets in the VANET.

When two nodes $i$ and $j$ meet, they first authenticate each other based on their certificates. Assume vehicle $i$ has a packet $M$ which is authorized to propagate, but node $j$ doesn't have that packet. Vehicle $i$ can forward the packet to $j$. Vehicle $j$ will also issue a receipt for that packet and send it to $i$. This receipt $E_{j}\left(H\left(M, P K_{i}\right)\right)$ serves as the evidence of $i$ 's spray.

$$
\begin{aligned}
& i \rightarrow j \quad: \quad M, E_{F A}(H(M, T)), T_{i}^{j}(M) \\
& j \rightarrow i \quad: \quad E_{j}\left(H\left(M, P K_{i}\right)\right), T_{j}^{i}(M)
\end{aligned}
$$

Only if the packet $M$ is received for the first time will a vehicle $j$ generate the receipt for $M . j$ should get a time-stamp from $i$ 's temper-proof device with $i$ 's signature as evidence to prove the time it initially stored the current packet. $j$ will also provide $i$ a time-stamp $T_{j}^{i}(M)$ generated by its temper-proof device in the receipt.

\section{Simulation}

\section{A. Simulation setup}

All simulations are carried out on a customized simulator. We set up the simulation in a $5,000 m \times 5,000 m$ area. We utilize the VANET mobility model [15], together with real data from the US Census Bureau's TIGER 2006 database (Palm Beach County, TGR12099) to generate the vehicles' mobility traces. The maximum number of vehicles in the network is 1,000 . We randomly select 50 of them to be source nodes. We randomly deploy the road side storage points at the intersections. The default transmission range is $50 \mathrm{~m}$. We assume each vehicle carries a $2 \mathrm{MB}$ buffer in our simulation studies, and packets will be deleted when the buffer is full. In all of the simulations, packets are $100 \mathrm{~KB}$ in size, and each signature is 128 bits. The default $\alpha=0.5, V_{r} / V=0.7$, $a=0.5$ and $p=0.5$.

For a vehicle $i$ in the forwarding tree, it will always store the packet until the buffer is full. When a vehicle $j$ without the current packet enters into $i$ 's communication range, $i$ will spray the packet to $j$ with probability 1 if the expected rewards surely increase; $i$ will spray the packet to $j$ with a low probability ( 0.5 in our simulation) if it is uncertain towards the reward increasing. We model the nodes outside the forwarding tree according to the cumulative prospect theory [16], which is a generally accepted model to describe how individuals evaluate small probability events, such as lotteries.

\section{B. Simulation results}

In Fig. 3, we examine the relationship between the average rewards with other parameters. Nodes are divided into different groups according to the selected parameter. For example, in Fig. 3(a), nodes are divided into groups according to their contribution, and the average reward for nodes whose contributions $C_{i} \in(0,5]$ are shown as the points above 5 .

Compared to the curve for proportional rewarding, the FRAME scheme appears to send greater rewards to nodes with the highest and lowest contributions in Fig. 3(a). For nodes with high contributions, they usually have more descents. The total contributions of the subtrees rooted at these nodes pushed their proportion of rewarding higher. For the nodes with low contributions, the average reward was increased due to the existence of the sweepstake component. For all three schemes, the average reward increased when contributions increased. Therefore, they all satisfy the fairness requirement.

As illustrated in Fig. 3(b), the FRAME scheme provides more reward than the proportional rewarding towards nodes that effectively sprayed the packet many times. This proves that the FRAME scheme has a better spray incentive than the proportional rewarding. Fig. 3(c) shows that the average reward in all schemes is not sensitive to the nodes' level in the tree (root is level 0). In this case, a node outside the tree will not discard the packet sprayed by a higher level node and wait for the invitation from the root.

Fig. 4(a) shows the delivery ratio in the unicast scenario, and the Fig. 4(b) shows the percentage of nodes that receive the packet in the broadcast scenario. The delivery ratio and penetration ratio when using the FRAME scheme are higher than in the other two methods. This proves the applicability of the FRAME scheme.

The drawback of the receipt counting scheme is shown in Fig. 5. We examine the total reward paid by the source in 


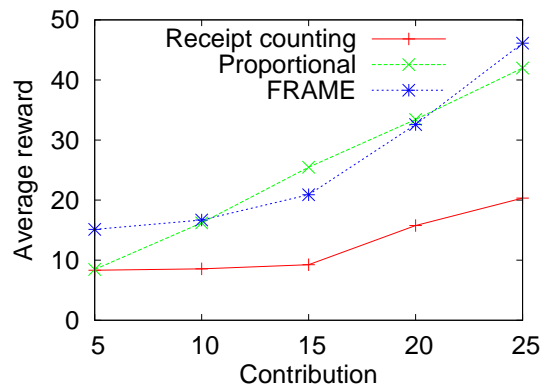

(a) Contribution

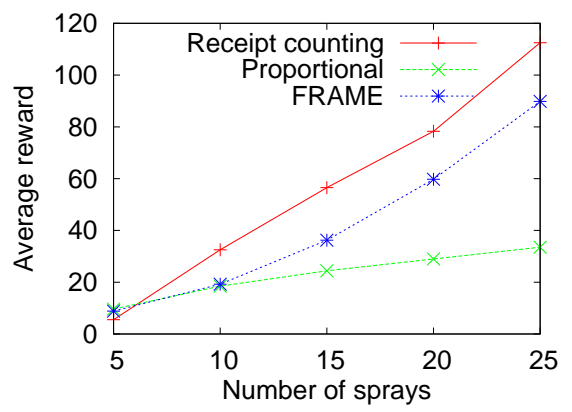

(b) Number of spray

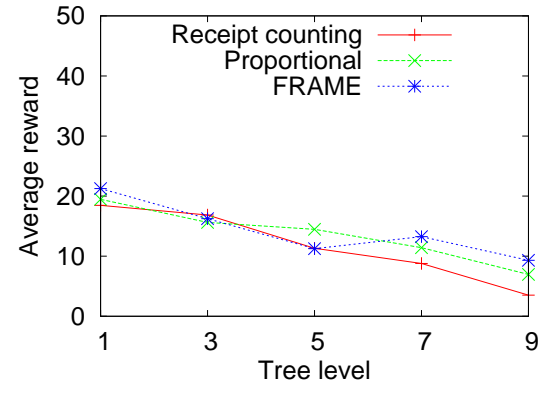

(c) Level

Fig. 3. Average reward comparison.

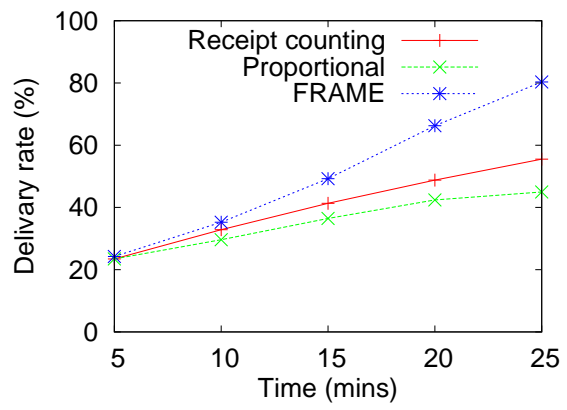

(a) Throughput

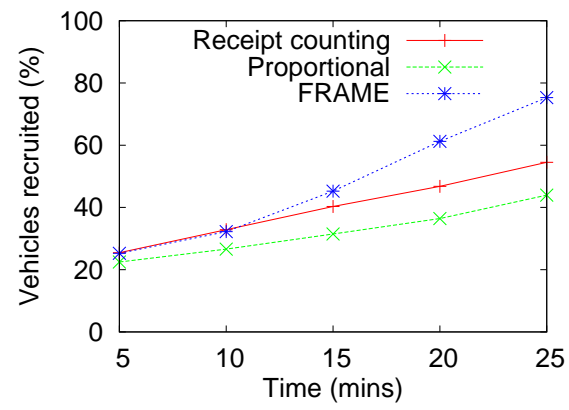

(b) Percentage of recruited nodes

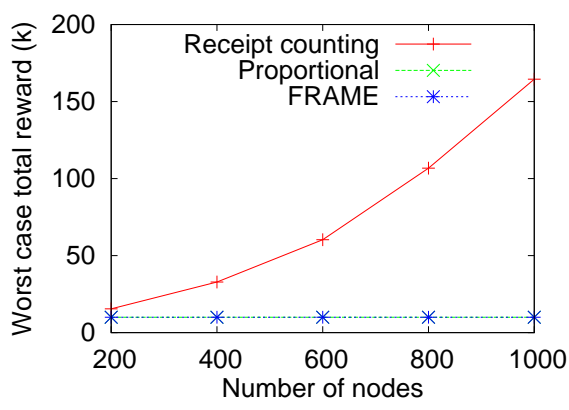

Fig. 5. Amount of overspending comparison.

Fig. 4. Traditional network measurement analysis.

the worst case. For proportional rewarding and the FRAME scheme, the totals are fixed. Although the average total reward remains acceptable, the worst case total reward may deter the source vehicles from sending out the packets when using the receipt counting scheme.

\section{CONCLUSION}

In this paper, we proposed a secure incentive scheme to motivate vehicles and their drivers to cooperate and contribute to the packet forwarding process in VANETs. We first defined the measurement of contribution according to the unique characteristics of VANET communication. We then proposed the weighted rewarding component that ensures fairness. We also presented the security measures as the basis for the incentive scheme. In the future, we plan to investigate incentive scheme that utilizes reputation instead of virtual credit in the VANETs.

\section{ACKNOWLEDGMENTS}

This work was supported in part by NSF grants CCR 0329741, CNS 0422762, CNS 0434533, CNS 0531410, and CNS 0626240. Email: fli4@fau.edu, jie@cse.fau.edu.

\section{REFERENCES}

[1] J. Luo and J. Hubaux. A Survey of Research in Inter-Vehicle Communications. pages 111-122, 2006.

[2] S. Lee, G. Pan, J. Park, M. Gerla, and S. Lu. Secure incentives for commercial ad dissemination in vehicular networks. In Proc. of ACM MobiHoc, 2007.
[3] A. Mislove, A. Post, K. Gummadi, and P. Druschel. Ostra: Leverging trust to thwart unwanted communication. In Proc. of ACM NSDI, 2008.

[4] J. Douceur and T. Moscibroda. Lottery trees: motivational deployment of networked systems. In Proc. of ACM SIGCOMM, 2007.

[5] C. Li, B. Yu, and K. Sycara. An incentive mechanism for message relaying in unstructured peer-to-peer systems. In Proc. of $A C M A A$ MAS, 2007.

[6] Q. Lian, Z. Zhang, Y. Peng, M. Yang, Y. Dai, and X. Li. Robust incentives via multi-level tit-for-tat. In Proc. of IPTPS, 2006

[7] T. Ngan, D. Wallach, and P. Druschel. Incentives-compatible peer-topeer multicast. In Proc. of Workshop on the Economics of Peer-to-Peer Systems, 2004.

[8] L. Buttyán and J. Hubaux. Stimulating Cooperation in Self-Organizing Mobile Ad Hoc Networks. Mobile Network Application, 8(5):579592, 2003.

[9] S. Zhong, L. Li, Y. Liu, and Y. Yang. On designing incentive-compatible routing and forwarding protocols in wireless ad-hoc networks: an integrated approach using game theoretical and cryptographic techniques. In Proc. of ACM MobiCom, 2005.

[10] P. Rogers. The Cognitive Psychology of Lottery Gambling: A Theoretical Review. Journal of Gambling Studies, 14(2):111-134, 1998.

[11] M. Raya and J. Hubaux. The security of vehicular ad hoc networks. In Proc. of Workshop on Security of ad hoc and sensor networks, 2005.

[12] B. Parno and A. Perrig. Challenges in securing vehicular networks. In Proc. of Workshop on Hot Topics in Networks, 2005.

[13] M. Raya, D. Jungels, P. Papadimitratos, I. Aad, and J. Hubaux. Certificate revocation in vehicular networks. In Technical Report LCA-Report2006-006, 2006

[14] D. Kahneman and A Tversky. Prospect Theory: An Analysis of Decision under Risk. Econometrica, 47(2):263-91, 1979.

[15] A. K. Saha and D. B. Johnson. Modeling mobility for vehicular ad-hoc networks. In Proc. of ACM VANET, 2004.

[16] A. Tversky and D. Kahneman. Advances in Prospect Theory: Cumulative Representation of Uncertainty. Journal of Risk and Uncertainty, 5(4):297-323, 1992 